By observing the motions of these peaks across the spectrograms, one may compute the locations of the topographic features which produced them. From each depolarized spectrum, the frequency deviation of each peak has been plotted. A reproduction of this plot is given in figure 5 . The solid curves represent locations on Venus which produce the best fit to the data, and the corresponding Venus-centered longitudes and latitudes are marked in the figure. The Venus rotation vector as described above was used in this computation. The fact that the theoretical curves fit the data so well indicates that the reflecting objects are, indeed, fixed to the planetary surface, and that an accurate rotation vector has been used.

Figure 6 describes the Venus-centered coordinate system. The North direction was chosen along Venus's rotation axis, but opposite to the usual direction since the rotation is retrograde. Thus, North is toward the celestial north pole. The prime meridian was chosen to go through the sharpest of the two features in the spectrograms. This meridiah contained the sub-Earth point on 23 July 1964. The positive direction in longitude is such that the sub-Earth point moves through successively larger angles. Figure 6 gives the locations of the "centers of reflection" of the two Venusian features, but the objects may be much larger than shown. Although the technique used gives a good indication of the longitudinal extent of the features, it gives little indication of their latitudinal extent.

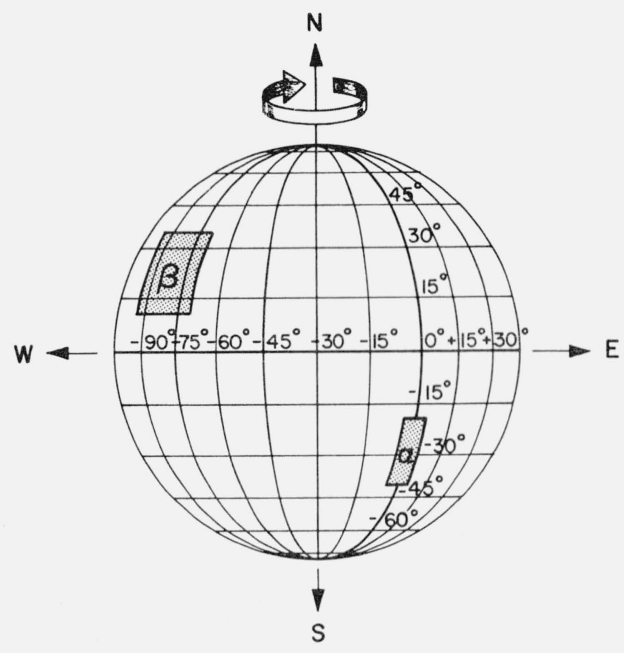

Figure 6. Venus-centered coordinate sysiem.

When the range-spectrum data has been reduced, additional features will be located in the equatorial belt of Venus.

\title{
Preliminary Mars Radar Results
}

\author{
Richard M. Goldstein
}

\section{Communications Systems Research Section, Jet Propulsion Laboratory, California Institute of Technology, Pasadena, Calif.}

This note presents some very preliminary results obtained by the Jet Propulsion Laboratory during its recent radar observations of Mars. These experiments were performed nearly every night of February, March, and half of April 1965. The radar parameters were the same as described in the previous paper in this same issue of Venus results, except the system temperature was lowered to $27^{\circ} \mathrm{K}$. However, since the power returned by Mars is less than Venus by a factor of almost 100, and Doppler broadening is greater by a factor of over 200, only spectral data was taken. That is, pure CW was transmitted for the $11 \mathrm{~min}$ time-of-flight, and then the echo was received for $11 \mathrm{~min}$. The received signal was then analyzed into its frequency spectrum.

Altogether, almost 1300 such runs were obtained, each covering a bandwidth of $3700 \mathrm{c} / \mathrm{s}$ with a frequency resolution of $84 \mathrm{c} / \mathrm{s}$. We note that the limb-to-limb Doppler broadening which Mars produces is $7670 \mathrm{c} / \mathrm{s}$. Thus, the small amount of power which is returned outside of the $3700-\mathrm{c} / \mathrm{s}$ bandwidth is not detected.

Although the power received in each run originates from most of the planetary disk, the largest portion of the power is returned from the sub-Earth point. Consequently, the runs were averaged according to the longitude of the corresponding sub-Earth points. The result is a set of 36 spectrograms, each the average of over 33 runs, which corresponds to $10^{\circ}$ increments of Martian longitude. The latitude of the sub-Earth point was close to $+21^{\circ}$ during the entire experiment.

${ }^{1}$ This paper presents the results of one phase of research carried out at the Jet Propulsion Laboratory, California Institute of Technology, under Contract No. NAS 7-100, sponsored by the National Aeronautics and Space Administration. 


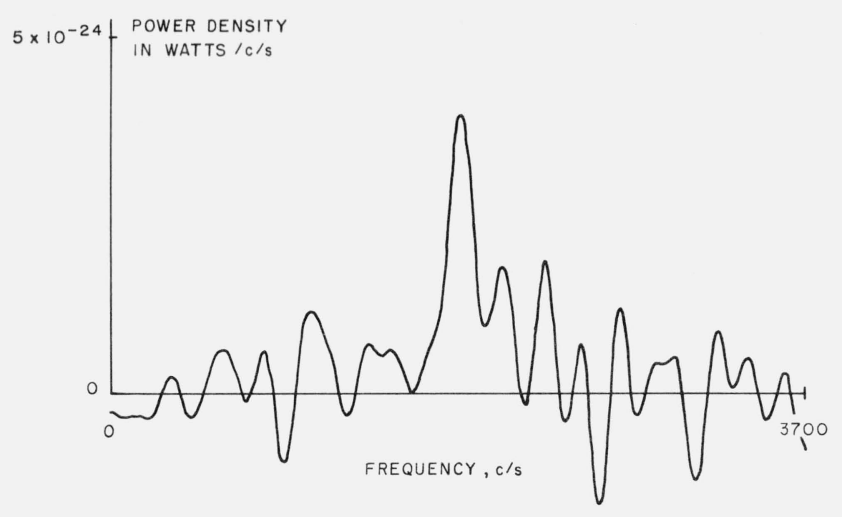

Figure 1. Mars spectrogram, $180^{\circ}$ to $190^{\circ}$ longitude.

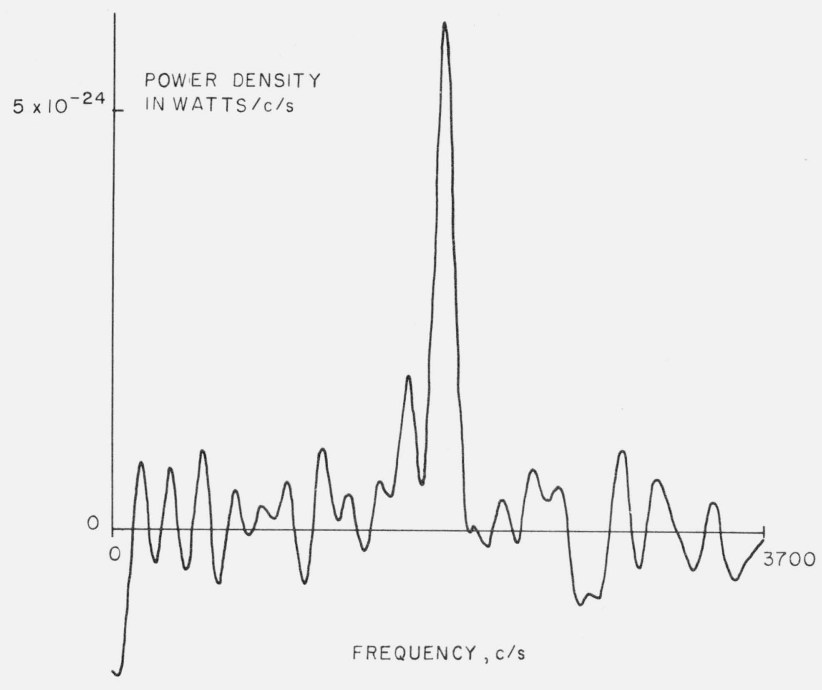

Figure 3. Mars spectrogram, $200^{\circ}$ to $210^{\circ}$ longitude.

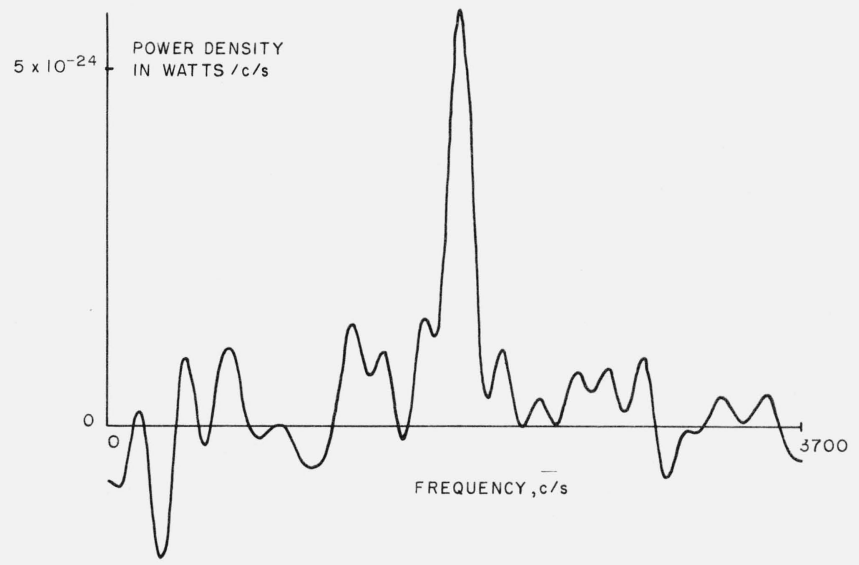

FigURE 2. Mars spectrogram, $190^{\circ}$ to $200^{\circ}$ longitude.

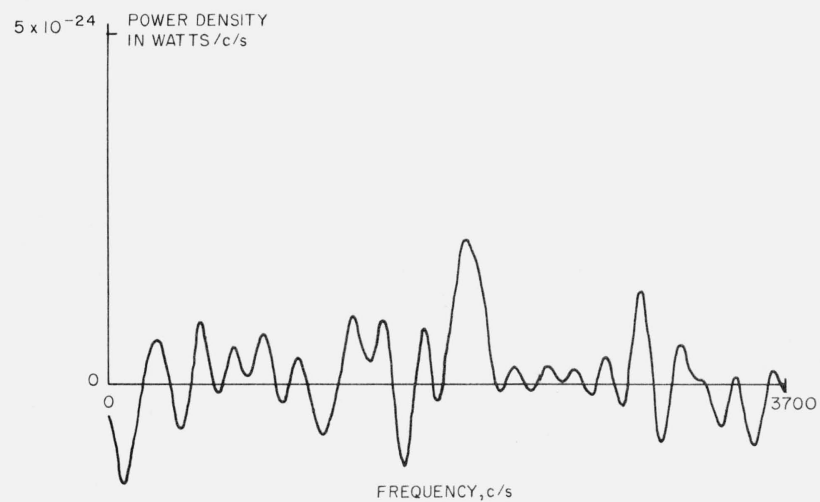

Figure 4. Mars $\begin{gathered}\text { spectrogram, } 210^{\circ} \text { to } 220^{\circ} \\ \text { longitude. }\end{gathered}$

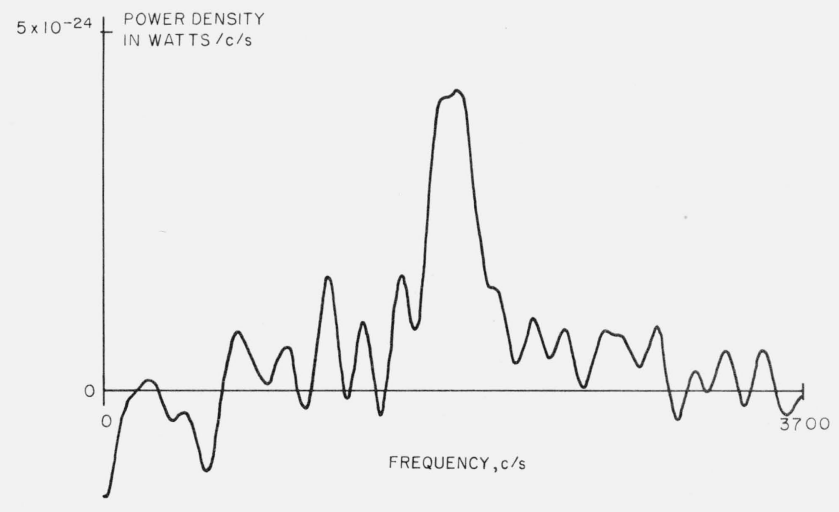

Figure 5. Mars spectrogram, $240^{\circ}$ to $250^{\circ}$ longitude. 
Most of these spectrograms show a small amount of power reflected diffusely by the disk. Some of them, however, show relatively strong, narrowband reflections, which originate from an area less than $2^{\circ}$ in extent about the sub-Earth point.

Figures 1 through 4 are samples of these spectrograms from successive $10^{\circ}$ strips of Mars. The narrowband echo is seen to increase, reaching a maximum at $200^{\circ}$ to $210^{\circ}$ (the region of Trivium Charontis), and then it drops off rather abruptly. One may conclude that there is a very smooth region on Mars, extending $20^{\circ}$ to $30^{\circ}$ in longitude, and of unknown latitudinal extent.

A similar, but wider band, sequence of echoes was reflected from the $240^{\circ}$ to $250^{\circ}$ region. Figure 5 is a sample spectrogram.

When data reduction is complete, both returned power and bandwidth will be presented as functions of longitude, and compared to the optical features on Mars. It may also be possible to show some of the regions of stronger reflectivity as functions of time as well.

(Paper 69D12-611)

\title{
Recent Ârecibo Observations of Mercury
}

\author{
G. H. Pettengill
}

Arecibo Ionospheric Observatory, ${ }_{1}^{1}$ Arecibo, Puerto Rico

As the new determination of Mercury's rotation is at variance with four generations of optical work and is not confirmed by JPL workers, it is appropriate to further discuss the Arecibo procedures.

Mercury moved into the Arecibo beam on 11 March 1965 with strongest echoes predicted for 7 April 1965. Although this conjunction should have given echo strengths slightly inferior to the conjunction of May 1964, improvements in receiver noise figure and data handling technique have more than made up for the less favorable geometry.

${ }^{1}$ Operated by Cornell University with the support of the Advanced Research Projects Agency under a research contract with the Air Force Office of Scientifi Research, OAR.

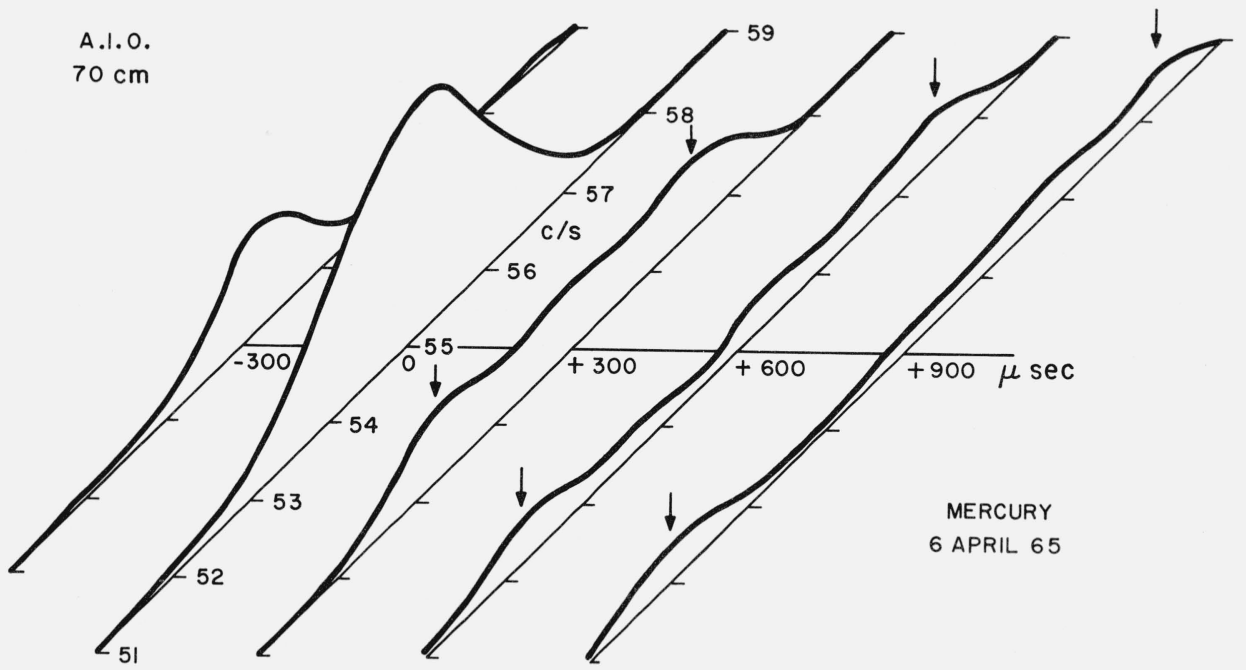

FIGURE 1. Plot of echo power as a function of relative delay (toward the right) and Doppler frequency shift (diagonally).

The arrows indicate the "wings", whose separation, when combined with the distance from the echo maximum, gives the instan taneous apparent rotation rate. 